\title{
Amine and guanidine emissions from a boreal forest floor Supplement
}

Marja Hemmilä ${ }^{1}$, Ulla Makkonen ${ }^{1}$, Aki Virkkula ${ }^{1,2}$, Georgia Panagiotopoulou ${ }^{1}$, Juho Aalto ${ }^{2}$, Markku Kulmala ${ }^{2}$, Tuukka Petäjä ${ }^{2}$, Hannele Hakola ${ }^{1}$, Heidi Hellén ${ }^{1}$

${ }^{1}$ Finnish Meteorological Institute, P.O. Box 503, 00101 Helsinki, Finland

${ }^{2}$ Insitute for Atmospheric and Earth System Research / Physics, Faculty of Science, University of Helsinki, Finland

Correspondence to: Marja Hemmilä (marja.hemmila@helsinki.fi)

Table ST1. Line equations (y) for chamber temperature - emission rate -plots. For DMA and guanidine the the $\mathbf{R}^{2}$ values were better, when temperatures were moved 2 hours above. For DMA best fit was with exponential function, and for DMA and guanidine with linear function.

\begin{tabular}{lccccc}
\hline \multicolumn{2}{c}{ DMA } & TMA & \multicolumn{2}{c}{ Guanidine } \\
\hline Date & $\mathrm{y}$ & $\begin{array}{c}\mathrm{y} \text { without } \\
\text { temperature } \\
\text { move }\end{array}$ & $\mathrm{y}$ & $\mathrm{y}$ & $\begin{array}{c}\text { y without } \\
\text { temperature } \\
\text { move }\end{array}$ \\
\hline 24.5. & $47.665 \mathrm{e}^{0.0406 \mathrm{x}}$ & $52.02 \mathrm{e}^{0.0353 \mathrm{x}}$ & $0.8477 \mathrm{x}+43.052$ & $0.8796 \mathrm{x}+34.74$ & $0.7096 \mathrm{x}+37.599$ \\
25.5. & $40.286 \mathrm{e}^{0.0443 \mathrm{x}}$ & $44.375 \mathrm{e}^{0.0388 \mathrm{x}}$ & $0.6173 \mathrm{x}+46.863$ & $0.9563 \mathrm{x}+30.149$ & $0.9791 \mathrm{x}+29.266$ \\
26.5. & $39.267 \mathrm{e}^{0.0445 \mathrm{x}}$ & $45.138 \mathrm{e}^{0.0376 \mathrm{x}}$ & $0.5484 \mathrm{x}+46.319$ & $0.8956 \mathrm{x}+31.927$ & $0.6978 \mathrm{x}+35.948$ \\
27.5. & $25.91 \mathrm{e}^{0.0328 \mathrm{x}}$ & $31.361 \mathrm{e}^{0.0234 \mathrm{x}}$ & $1.4679 \mathrm{x}-7.7149$ & $1.4101 \mathrm{x}-0.652$ & $1.4651 \mathrm{x}-1.203$ \\
18.7. & $103.17 \mathrm{e}^{0.0596 \mathrm{x}}$ & $196.89 \mathrm{e}^{0.0371 \mathrm{x}}$ & $9.5789 \mathrm{x}-140.3$ & $1.0618 \mathrm{x}+18.844$ & $0.5088 \mathrm{x}+34.539$ \\
19.7. & $77.778 \mathrm{e}^{0.0624 \mathrm{x}}$ & $120.57 \mathrm{e}^{0.0454 \mathrm{x}}$ & $10.758 \mathrm{x}-154.65$ & $0.6528 \mathrm{x}+29.577$ & $0.5907 \mathrm{x}+31.155$ \\
20.7. & $84.551 \mathrm{e}^{0.0396 \mathrm{x}}$ & $89.87 \mathrm{e}^{0.0371 \mathrm{x}}$ & $4.7532 \mathrm{x}-40.486$ & $0.2202 \mathrm{x}+38.772$ & $0.2837 \mathrm{x}+37.317$ \\
21.7. & $177.1 \mathrm{e}^{-0.047 \mathrm{x}}$ & $92.12 \mathrm{e}^{-0.016 \mathrm{x}}$ & $-0.3408 \mathrm{x}+43.364$ & $-1.1165 \mathrm{x}+61.042$ & $-0.2303 \mathrm{x}+42.245$ \\
\hline
\end{tabular}



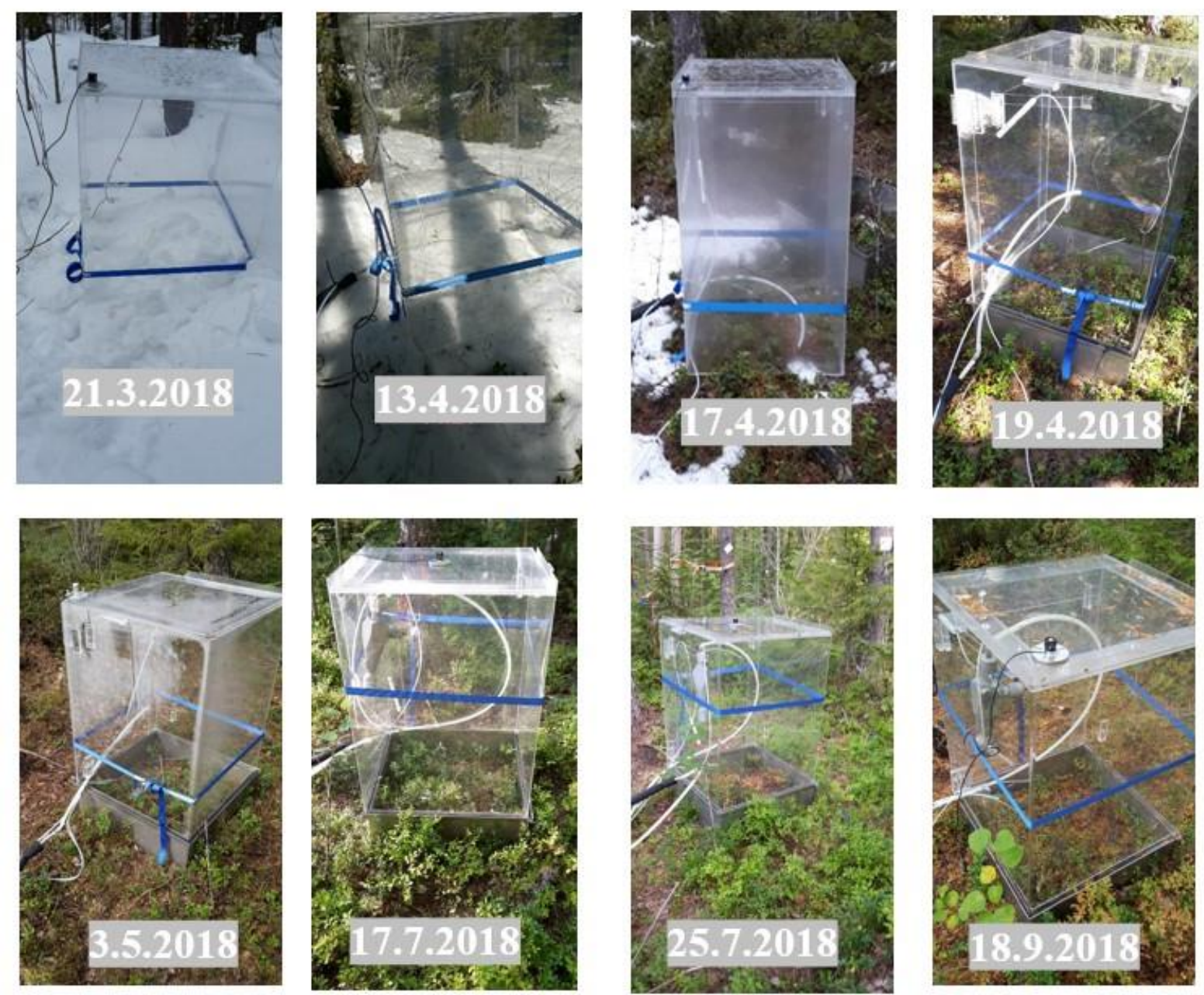

Figure S1. The chamber in different measurement periods. 